\title{
Solvable Artin representations ramified at one prime
}

\author{
Jonah Leshin
}

October 12, 2018

\begin{abstract}
We classify the possibilities for the fixed field of the kernel of an irreducible threedimensional Artin representation of $\mathbb{Q}$ with solvable image ramified at one prime by using the classification of the finite irreducible subgroups of $\mathrm{PGL}_{3}(\mathbb{C})$. This allows us to bound the number of such representations with given Artin conductor.
\end{abstract}

\section{Introduction}

The Langlands program gives a correspondence between Galois representations and automorphic representations. In the case of two-dimensional Artin representations, the corresponding analytic objects are modular forms of weight one and Maass forms of weight zero, which correspond to odd and even two-dimensional Artin representations, respectively. Specifically, Deligne and Serre [3] showed that given a cuspidal modular form of weight 1 , level $N$, and Nebentypus character $\epsilon$ with normalized $\left(a_{1}=1\right)$ Fourier expansion $\sum a_{n} q^{n}$, there exists an Artin representation

$$
\rho: \mathrm{G}_{\mathbb{Q}}:=\operatorname{Gal}(\overline{\mathbb{Q}} / \mathbb{Q}) \rightarrow \mathrm{GL}_{2}(\mathbb{C})
$$

of conductor $N$ such that (via $\rho$ ) the trace of the conjugacy class of the Frobenius at $p$, Frob , in $\mathrm{G}_{\mathbb{Q}}$ is $a_{p}$, and the determinant of Frob ${ }_{p}$ is $\epsilon(p)$. Conversely, given a two-dimensional Artin representation $\rho$, if we insist that either $\rho$ has solvable image [1] or that $\rho$ is odd [13], then there is known to exist an automorphic form $f$ such that $f$ gives rise to $\rho$ in the manner described above. Using this correspondence, Wong [20], building on work of Duke [5], bounded the number of two-dimensional Artin representations of $\mathbb{Q}$ with Artin conductor $N$ in terms of $N$.

Less is known in the case of three-dimensional Artin representations. In this paper we bound the number of three-dimensional irreducible Artin representations with solvable image and prime power conductor. It is known that such representations come from automorphic representations of $\mathrm{GL}_{3}\left(\mathbb{A}_{\mathbb{Q}}\right)$, where $\mathbb{A}_{\mathbb{Q}}$ denotes the adèles of $\mathbb{Q}$. We use purely algebraic techniques for our bounds, which therefore, in principle, could give bounds for the number of automorphic representations of $\mathrm{GL}_{3}\left(\mathbb{A}_{\mathbb{Q}}\right)$ with particular properties.

The main results of this paper, which are made more precise in Theorems 1, 2, 4, and 5 are: 
- The number of irreducible, imprimitive three-dimensional Artin representations of $\mathrm{G}_{\mathbb{Q}}$ with conductor dividing $p^{m}$ is at most $C m p^{m+1}$, where $C$ is an explicitly given constant.

- The number of solvable, irreducible, primitive three-dimensional Artin representations of $\mathrm{G}_{\mathbb{Q}}$ with conductor dividing $p^{m}$ is at most $D p^{\frac{m}{3}+50}$, where $D$ is an explicitly given constant.

The paper is divided into two main parts. The first part looks at imprimitive representations. We use the fact that any imprimitive three-dimensional representation is monomial to bound the number of all imprimitive representations in one fell swoop. The second part deals with primitive representations, where we do a case by case analysis of the three groups that can occur as the projective image of a primitive three-dimensional Artin representation.

\section{Definitions and preliminaries}

Let $V$ be a finite dimensional vector space over an arbitrary field, and let $G$ be a group. A representation $\rho: G \rightarrow \mathrm{GL}(V)$ is called imprimitive if there exist proper subspaces $V_{1}, \ldots, V_{m}$ of $V$ such that $V=\oplus_{i=1}^{m} V_{i}$ and $\rho(G)$ permutes the $V_{i}$. If such a decomposition of $V$ does not exist, $\rho$ is called primitive. If $\rho$ is imprimitive, and if the corresponding $V_{i}$ are one-dimensional and permuted transitively by $G$, then $\rho$ is said to be monomial (this is equivalent to the standard definition of monomial, which is that there exists a subgroup $H \leq G$ and a linear character $\chi$ of $H$ such that $\rho$ is induced from $\chi$ ). Thus monomial representations of dimension greater than 1 are imprimitive, and the converse is true if the dimension of the representation is prime, and if $G$ acts transitively on the $V_{i}$. A group is said to be monomial if each of its irreducible representations is monomial. A comprehensive treatment of these definitions and the general theory of representations of finite groups can be found in [2].

An Artin representation of a number field $F$ is a continuous homomorphism

$$
\rho: \operatorname{Gal}(\bar{F} / F) \rightarrow \mathrm{GL}_{n}(\mathbb{C}) .
$$

We take the analytic topology on $\mathrm{GL}_{n}(\mathbb{C})$, so Artin representations necessarily have finite image. The conductor $N(\rho)$ of $\rho$ is defined to be the product of its local conductors, whose definition we now recall. Let $\mathfrak{p}$ be a prime of $F$. For the $\mathfrak{p}$-part $N_{\mathfrak{p}}(\rho)$ of $N(\rho)$, consider the restriction $\rho_{\mathfrak{p}}$ of $\rho$ to $\mathrm{G}\left(\bar{F}_{\mathfrak{p}} / F_{\mathfrak{p}}\right)$ (if $E / F$ is an extension of fields, we will write $\mathrm{G}(E / F)$ in place of $\operatorname{Gal}(E / F)$ to ease notation). The representation $\rho_{\mathfrak{p}}$ factors through a finite quotient $\mathrm{G}\left(K / F_{\mathfrak{p}}\right)$ of $\mathrm{G}\left(\bar{F}_{\mathfrak{p}} / F_{\mathfrak{p}}\right)$. Let $g_{i}$ denote the order of the $i$ th ramification group $G_{i}$ of $\mathrm{G}\left(K / F_{\mathfrak{p}}\right)$. We then define $N_{\mathfrak{p}}(\rho)$ to be $\mathfrak{p}$ raised to the power

$$
\sum_{i \geq 0} \frac{g_{i}}{g_{0}} \operatorname{dim} V / V^{G_{i}},
$$

where $V$ is the representation space for $\rho$ and $V^{G_{i}}$ is the subspace of elements fixed by $G_{i}$. If $\mathfrak{a}$ is an ideal in the ring of integers $\mathfrak{O}$ of a number field, such as the conductor of an Artin 
representation, and if $\mathfrak{p}$ is a prime of $\mathfrak{O}$, we take $v_{\mathfrak{p}}(\mathfrak{a})$ to be the exponent of the highest power of $\mathfrak{p}$ dividing $\mathfrak{a}$. For the remainder of this paper we make the following hypothesis on all Artin representations:

$$
F=\mathbb{Q} \text { and } n=3 .
$$

Representations will always be distinguished up to isomorphism. The natural projection $\pi: \mathrm{GL}_{3}(\mathbb{C}) \rightarrow \mathrm{PGL}_{3}(\mathbb{C})$ will always be denoted by $\pi$, and a lift of a projective representation $\psi: G \rightarrow \mathrm{PGL}_{3}(\mathbb{C})$ of a group $G$ is any representation $\rho: G \rightarrow \mathrm{GL}_{3}(\mathbb{C})$ such that $\psi=\pi \circ \rho$. A cyclic group of order $n$ will be denoted by $C_{n}$, and $Z(G)$ denotes the center of a group $G$. If $L / K$ is a Galois extension of fields, we say $E$ is a central extension of $L / K$ if $E / K$ is Galois and $\mathrm{G}(E / L) \leq Z(\mathrm{G}(E / K))$.

The symbol $E_{\rho}$ will denote the fixed field of the kernel of one of our irreducible threedimensional Artin representations $\rho$ with solvable image, and $F_{\rho}$ will denote the fixed field of the kernel of $\pi \circ \rho$ (when the context is clear, the subscript $\rho$ may be dropped). Thus $\rho$ descends to an injection $\mathrm{G}\left(E_{\rho} / \mathbb{Q}\right) \hookrightarrow \mathrm{GL}_{3}(\mathbb{C})$, which by abuse of notation we also denote by $\rho$, and $Z(\mathrm{G}(E / \mathbb{Q}))=\mathrm{G}(E / F)$, which is cyclic since $\rho$ is irreducible (by Schur's Lemma [18, Section 2.2]).

We say an Artin representation $\rho$ is ramified at $p$ if the extension $E_{\rho} / \mathbb{Q}$ is ramified at $p$. In sections 3 and 4, all representations are assumed to be unramified outside $p \infty$, where $p$ is a finite prime and $\infty$ is the real place of $\mathbb{Q}$. To avoid special cases, most of which are trivial, we will assume $p \neq 2,3$.

In our computations, we will wish to bound the number of $C_{a}$ or $C_{a} \times C_{a}$ extensions of a number field $K$ with given ramification conditions, where $a$ is prime. We do this by first finding the $a$-rank of the maximal extension $L$ of $K$ with the given ramification conditions. Let $r_{a, b}(x)$ be the number of $\left(C_{a}\right)^{b}$ extensions of $K$ contained in a Galois extension $L$, where $\mathrm{G}(L / K)$ is abelian and has $a$-rank $x$. We will consider the cases when $a=2$ or 3 , although the following formulas hold for any prime $a$ :

$$
r_{a, 1}(x)=\frac{a^{x}-1}{a-1}, \quad r_{a, 2}(x)=\frac{\left(a^{x}-1\right)\left(a^{x}-a\right)}{\left(a^{2}-1\right)\left(a^{2}-a\right)} .
$$

When we have an extension $L / K$ of number fields with $L / \mathbb{Q}$ and $K / \mathbb{Q}$ Galois, and $L / \mathbb{Q}$ ramified only at $p$, we will write $e(L / K)$ to denote the ramification from $K$ to $L$ of the primes above $p$ in $K$. We will say an extension of fields is unramified if it is unramified at all finite primes. The ring of integers of a number field $K$ will be denoted by $\mathfrak{O}_{K}$ and its absolute discriminant by $d_{K}$. We let $h_{K}$ denote the class number of $K, \mathrm{Cl}(K)$ the ideal class group of $K, \mathrm{Cl}^{\mathfrak{m}}(K)$ the ray class group of $K$ of modulus $\mathfrak{m}, H_{K}$ the Hilbert class field of $K$, and $K^{\mathfrak{m}}$ the ray class field of $K$ of modulus $\mathfrak{m}$. If $\mathfrak{m}$ is a product of finite primes of $K$, we will write $\mathfrak{m} \infty$ to denote the modulus that is the product of $\mathfrak{m}$ multiplied by all the real places of $K$. 


\section{Imprimitive Representations}

In this section we assume that $\rho: \mathrm{G}_{\mathbb{Q}} \rightarrow \mathrm{GL}_{3}(\mathbb{C})$ is irreducible, imprimitive, and unramified outside $p \infty$. Since $\rho$ is monomial, there exists a field $L$ of degree 3 over $\mathbb{Q}$ and a character $\chi: \mathrm{G}_{L} \rightarrow \mathbb{C}^{*}$ such that $\rho=\chi_{L}^{\mathbb{Q}}$, where $\chi_{L}^{\mathbb{Q}}$ denotes the induced representation of $\chi$ to $\mathrm{G}_{\mathbb{Q}}$.

Suppose first that $L \subset \mathbb{Q}\left(\zeta_{p}\right)$ and let $\mathfrak{p}$ be the prime of $L$ above $p$. It follows from the Corollary to Proposition 4, Chapter VI of [19] that

$$
v_{p}(N(\rho))=v_{\mathfrak{p}}(N(\chi))+2
$$

Let

$$
\begin{gathered}
c_{m}=\#\left\{\text { Irreducible } \rho: \mathrm{G}_{\mathbb{Q}} \rightarrow \mathrm{GL}_{3}(\mathbb{C}) \text { such that } N(\rho) \mid p^{m} \text { and there exists } \chi: \mathrm{G}_{L} \rightarrow \mathbb{C}^{*}\right. \\
\left.\quad \text { such that } \rho=\chi_{L}^{\mathbb{Q}}\right\} \\
d_{m}=\#\left\{\chi: \mathrm{G}_{L} \rightarrow \mathbb{C}^{*} \text { such that } N(\chi) \mid \mathfrak{p}^{m}\right\} .
\end{gathered}
$$

It follows from Mackey's irreducibility criterion [18, Section 7.4] that the induction $\rho$ to $\mathrm{G}_{\mathbb{Q}}$ of a non-trivial $\chi \in \operatorname{Hom}\left(\mathrm{G}_{L}, \mathbb{C}^{*}\right)$ will be irreducible exactly when $\rho$ restricted to $\mathrm{G}_{L}$ is the sum of three distinct characters, each of which induces to give $\rho$ by Frobenius reciprocity [18, Section 7.2]. Therefore, using (1), we have

$$
c_{m} \leq \frac{d_{m-2}}{3}
$$

We first bound $d_{m}$. By class field theory, any $\chi$ with $N(\chi) \mid \mathfrak{p}^{m}$ factors through $\mathrm{G}\left(L^{\mathfrak{p}^{m}} / L\right)$, where $L^{\mathfrak{p}^{m}}$ is the ray class field of $L$ of modulus $\mathfrak{p}^{m}$. Class field theory gives the exact sequence

$$
\mathfrak{O}_{L}^{*} \rightarrow\left(\mathfrak{O}_{L} / \mathfrak{p}^{m} \infty\right)^{*} \rightarrow \mathrm{Cl}^{\mathfrak{p}^{m} \infty}(L) \rightarrow \mathrm{Cl}(L) \rightarrow 1
$$

where $\left(\mathfrak{O}_{L} / \mathfrak{p}^{m} \infty\right)^{*}$ is defined to be $\left(\mathfrak{O}_{L} / \mathfrak{p}^{m}\right)^{*} \times\langle \pm 1\rangle^{3}$, the exponent 3 coming from the 3 real places of $L$. The image of $\mathfrak{O}_{L}^{*}$ in $\left(\mathfrak{O}_{L} / \mathfrak{p}^{m} \infty\right)^{*}$ has order at least two, so we obtain

$$
d_{m}=\left|\mathrm{G}\left(L^{\mathfrak{p}^{m} \infty} / L\right)\right| \leq 4 h_{L} p^{m-1}(p-1)<4\left(\frac{1}{2} p\right) p^{m-1}(p-1)=2 p^{m}(p-1),
$$

where the first inequality follows from (2), and the second inequality from [14, Corollary 4], for which we need the fact that $L$ is totally real. Thus we obtain

Theorem 1. Notation as above,

$$
c_{m}<\frac{2 p^{m-1}}{3}
$$

For the remainder of this section, suppose that $L \nsubseteq \mathbb{Q}\left(\zeta_{p}\right)$, or equivalently, that $L / \mathbb{Q}$ is not Galois. By the following lemma, $p$ must split in $L$ as $\mathfrak{p}_{1}^{2} \mathfrak{p}_{2}$.

Lemma 1. Let $L / \mathbb{Q}$ be a degree 3 extension ramified at a single finite prime $p \neq 2,3$. Then $L / \mathbb{Q}$ is Galois if and only if $p$ is totally ramified in $L$. 
Proof. The only if part is clear. For the converse, suppose $L / \mathbb{Q}$ is totally ramified at $p$ and let $\mathfrak{p}$ be the prime of $L$ above $p$. Let $L_{\mathfrak{p}}$ be the completion of $L$ at $\mathfrak{p}$ and $\mathfrak{O}=\mathfrak{O}_{L_{\mathfrak{p}}}$ the ring of integers in $L_{\mathfrak{p}}$. Let $f(x)$ be the minimal polynomial for $\alpha \in \mathfrak{O}$, where $\alpha$ generates $\mathfrak{O}$ as a $\mathbb{Z}_{p}$-algebra. We may assume $\alpha \in \mathfrak{O}_{L}$. The $p$-part of the discriminant $\operatorname{disc}(f)$ of $f$ equals the local discriminant $d_{L_{\mathfrak{p}} / \mathbb{Q}_{p}}$, which has $p$-part $p^{2}(p \neq 3)$, the same as the $p$-part of the global discriminant $d_{L / \mathbb{Q}}$. So we can write $\operatorname{disc}(f)=\epsilon p^{2} D$, with $\epsilon \in\{ \pm 1\}$ and $p \nmid D$. The Galois closure $K$ of $L / \mathbb{Q}$ is ramified only at $p$ and is given by $K=L(\sqrt{\operatorname{disc}(f)})=L(\sqrt{\epsilon D})$. For $p \neq 2$, this is only possible if $\sqrt{\epsilon D} \in L$, i.e., $L / \mathbb{Q}$ is Galois.

By the Corollary to Proposition 4 of [19], we have

$$
N\left(\chi_{L}^{\mathbb{Q}}\right)=N_{L / \mathbb{Q}}(N(\chi)) d_{L}
$$

It follows that if $N(\chi)=\mathfrak{p}_{1}^{a_{1}} \mathfrak{p}_{2}^{a_{2}}$, then $N(\rho)=p^{a_{1}+a_{2}} p$. Let $c_{m}=c_{m, L}$ be the number of imprimitive $\rho$ with conductor dividing $p^{m}$ that are induced from $\mathrm{G}_{L}$. To estimate $c_{m}$, we need to estimate

$$
\#\left\{\chi: N(\chi) \mid \mathfrak{p}_{1}^{a_{1}} \mathfrak{p}_{2}^{a_{2}}, a_{1}+a_{2} \leq m-1\right\} .
$$

Thus we need to bound $\sum_{a_{1}+a_{2}=m-1}\left|\mathrm{G}\left(L^{\mathfrak{p}_{1}^{a_{1}} \mathfrak{p}_{2}^{a_{2}} \infty} / L\right)\right|$. We have

$$
c_{m} \leq \sum_{a_{1}+a_{2}=m-1}\left|\mathrm{G}\left(L^{\mathfrak{p}_{1}^{a_{1}} \mathfrak{p}_{2}^{a_{2}} \infty} / L\right)\right| \leq 4 h_{L} \sum_{a_{1}+a_{2}=m-1}\left(p^{a_{1}}-1\right)\left(p^{a_{2}}-1\right)<4 h_{L} m p^{m-1}
$$

where the first inequality follows from the exact sequence (2) applied to the situation at hand.

Since $L / \mathbb{Q}$ is not Galois, $\mathrm{G}_{L}$ is not normal in $\mathrm{G}_{\mathbb{Q}}$, so it is possible that there may only be one $\chi \in \operatorname{Hom}\left(\mathrm{G}_{L}, \mathbb{C}^{*}\right)$ that gives $\rho$ when induced up to $\mathrm{G}_{\mathbb{Q}}$. Since we are assuming that $H:=\mathrm{G}\left(E_{\rho} / L\right)$ is not normal in $G:=\mathrm{G}\left(E_{\rho} / \mathbb{Q}\right)$, the group $H$ has three conjugate subgroups inside $G$. Given a coset $s H$ and $\chi \in \operatorname{Hom}\left(H, \mathbb{C}^{*}\right)$, we can define the representation $\chi^{s} \in \operatorname{Hom}\left(s H s^{-1}, \mathbb{C}^{*}\right)$ by $\chi^{s}(x)=\chi\left(s^{-1} x s\right)$. The formula for an induced character [18, Section 7.2] shows that $\chi^{s}$ induced up to $G$ is isomorphic to $\chi$ induced up to $G$. The three conjugate subgroups $H^{s}$ correspond to conjugates of $L$, each degree three over $\mathbb{Q}$. Thus, it remains to bound $h_{L}$ and $\frac{n_{p}}{3}$, where $n_{p}$ is the number of non-Galois fields $L$ of degree 3 over $\mathbb{Q}$, ramified only at $p \infty$. We do the latter first. Let $L$ be such a number field. The Galois closure $K$ of $L / \mathbb{Q}$ is an $S_{3}$ extension of $\mathbb{Q}$ ramified only at $p \infty$. By Lemma $\mathbb{1}$, $p$ splits as $\mathfrak{p}_{1}^{2} \mathfrak{p}_{2}$ in $L$. Let $\mathfrak{q}$ be a prime of $K$ above $\mathfrak{p}_{2}$. We have

$$
e(\mathfrak{q} / p)=e\left(\mathfrak{q} / \mathfrak{p}_{2}\right) e\left(\mathfrak{p}_{2} / p\right)=e\left(\mathfrak{q} / \mathfrak{p}_{2}\right) \leq 2 .
$$

For any primes $\mathfrak{q}_{i}, \mathfrak{q}_{j}$ of $K$ above $p, e\left(\mathfrak{q}_{i} / p\right)=e\left(\mathfrak{q}_{j} / p\right)$, and the quantity is at least 2 , so by (4), it equals 2. Thus $K / \mathbb{Q}\left(\sqrt{p^{*}}\right)$ is unramified, where $\mathbb{Q}\left(\sqrt{p^{*}}\right)$ is the subfield of $K$ of degree 2 over $\mathbb{Q}$. Therefore, $L$ and its two Galois conjugates contained in $K$ combine to give one unramified $C_{3}$ extension of $\mathbb{Q}\left(\sqrt{p^{*}}\right)$. By [15, page 95$]$, we have $h_{\mathbb{Q}\left(\sqrt{p^{*}}\right)}<\frac{22.2 p}{\pi^{2}}$. Ramification at the infinite primes of $\mathbb{Q}\left(\sqrt{p^{*}}\right)$ can only contribute a power of 2 to the degree of the maximal abelian unramified (at all finite primes) extension of $\mathbb{Q}\left(\sqrt{p^{*}}\right)$. Since we are 
interested in the 3-rank of this extension, we may ignore the potential ramification at the infinite primes. We have the obvious bound that the 3 -rank of $\mathrm{Cl}\left(\mathbb{Q}\left(\sqrt{p^{*}}\right)\right)$ is less than

$$
\log _{3}\left(\frac{22.2 p}{\pi^{2}}\right)
$$

from which it follows that

$$
\frac{n_{p}}{3}<r_{3,1}\left(\log _{3}\left(\frac{22.2 p}{\pi^{2}}\right)\right)
$$

which is less than

$$
\frac{11.1 p}{\pi^{2}}
$$

Putting this last bound together with (3) , and using the bound $h_{L}<\frac{22.2 p}{\pi^{3}}$ (which follows from [15, page 95]), we obtain

Theorem 2. The number of imprimitive $\rho$ with $\rho=\chi_{L}^{\mathbb{Q}}, N(\rho)$ dividing $p^{m}$, and L/Q nonGalois is less than

$$
\left(\frac{985.7 m p^{m+1}}{\pi^{5}}\right) \text {. }
$$

Remark 1. It is possible to obtain a slightly better bound by using a slightly better bound for the 3-rank of $\mathrm{Cl}\left(\mathbb{Q}\left(\sqrt{p^{*}}\right)\right)$. See, for example, [16].

\section{Primitive representations}

For this section, suppose that $\rho: \mathrm{G}_{\mathbb{Q}} \rightarrow \mathrm{GL}_{3}(\mathbb{C})$ is primitive. We continue with our assumption that $\rho$ is unramified outside $p \infty$ and has solvable image. Since $\rho$ is primitive with solvable image, the image of $\pi \circ \rho$ in $\mathrm{PGL}_{3}(\mathbb{C})$ is isomorphic to one of the following groups [4, Theorem 4.8]:

$$
P_{1}:=\left(C_{3} \times C_{3}\right) \rtimes C_{4}, \quad P_{2}:=\left(C_{3} \times C_{3}\right) \rtimes Q_{8}, \quad P_{3}:=\left(C_{3} \times C_{3}\right) \rtimes \mathrm{SL}_{2}\left(\mathbb{F}_{3}\right),
$$

where $Q_{8}$ is the quaternion group of order 8 . We can view $P_{1}$ as a subgroup of $P_{2}$, and $P_{2}$ as a subgroup of $P_{3}$.

We claim that $\operatorname{Im}(\pi \circ \rho)$ cannot be $P_{2}$. If $\mathrm{G}\left(F_{\rho} / \mathbb{Q}\right)=P_{2}$, then $F_{\rho}$ (and thus $E_{\rho}$ ) has a subfield $L$ with $L / \mathbb{Q}$ Galois and $\mathrm{G}(L / \mathbb{Q}) \cong C_{2} \times C_{2}$. But there is no such field ramified only at one prime $(p \neq 2)$ of $\mathbb{Q}$.

In the next two subsections, we consider the cases $\mathrm{G}\left(F_{\rho} / \mathbb{Q}\right)=P_{1}$ and $\mathrm{G}\left(F_{\rho} / \mathbb{Q}\right)=P_{3}$. For each case, we first fix a number field $F$ with $\mathrm{G}(F / \mathbb{Q})=P_{i}$ and bound the number of representations $\rho$ with $F_{\rho}=F$. We then bound the number of such possible $F$-i.e., $F$ such that $\mathrm{G}(F / \mathbb{Q})=P_{i}$ and $F / \mathbb{Q}$ is unramified outside $p \infty$.

We will use the fact that the Schur multiplier $H_{2}(G, \mathbb{Z})=C_{3}$ for $G=P_{1}$ and $G=P_{3}$. An alternative definition of the Schur multiplier of a group $G$ is the largest group $X$ such that there exists a group $\Gamma$ with $\Gamma / X \cong G$ and $X \subseteq \Gamma^{\prime} \cap Z(\Gamma)$ [12, page 151], where $\Gamma^{\prime}$ is the 
commutator subgroup of $\Gamma$. Using this definition, one shows that the $p$-Sylow subgroup of $H_{2}(G, \mathbb{Z})$ is isomorphic a subgroup of the Schur multiplier of a $p$-Sylow subgroup of $G$. Using this fact, it is an exercise to show that $H_{2}\left(P_{1}, \mathbb{Z}\right)=C_{3}$. To show that $H_{2}\left(P_{3}, \mathbb{Z}\right)=C_{3}$, we need to show that the Sylow-2 subgroup of $P_{3}$, the quaternion group $Q_{8}$ of order 8 , has trivial Schur multiplier and that the Sylow-3 subgroup $\left(C_{3} \times C_{3}\right) \rtimes C_{3}$ of $P_{3}$ has Schur multiplier $C_{3}$. The former holds because every abelian subgroup of $Q_{8}$ is cyclic, and one can show that all such group have trivial Schur multiplier. To show the latter, one can, for example, use the facts about the Schur multiplier of a semi-direct product that are proven in [6].

\subsection{Representations with projective image isomorphic to $P_{1}$}

We count the number of Artin representations $\rho$ unramified outside $p \infty$ with projective image isomorphic to $P_{1}$, i.e., $\mathrm{G}\left(F_{\rho} / \mathbb{Q}\right) \cong P_{1}$. Let $L$ denote the fixed field of $C_{3} \times C_{3} \leq P_{1}$. Since $L$ is unramified outside $p \infty$, it is the unique degree four subfield of $\mathbb{Q}\left(\zeta_{p}\right)$. Writing $\left[E_{\rho}: F_{\rho}\right]=n$, we have the following tower of fields.

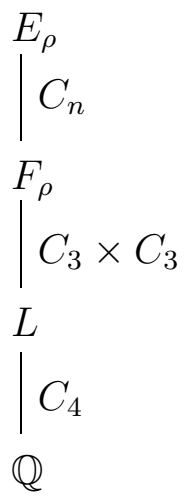

Lemma 2. Let $K$ be a subfield of $\mathbb{Q}\left(\zeta_{p}\right)$, and let $\mathfrak{p}$ be the unique prime of $K$ above the rational prime $p$. Then $K^{\mathfrak{p} \infty}=H_{K}\left(\zeta_{p}\right)$.

Proof. Certainly $H_{K}\left(\zeta_{p}\right) \subseteq K^{\mathfrak{p} \infty}$. Suppose the containment is strict. Then $K^{\mathfrak{p} \infty} / H_{K}\left(\zeta_{p}\right)$ is totally ramified at the primes of $H_{K}\left(\zeta_{p}\right)$ above $p$. It follows that $K^{\mathfrak{p} \infty} / \mathbb{Q}\left(\zeta_{p}\right)$ is tamely ramified at the prime $\left(1-\zeta_{p}\right)$ of $\mathbb{Q}\left(\zeta_{p}\right)$ above $p$. But such an extension cannot exist since $\mathbb{Q}\left(\zeta_{p}\right)^{\left(1-\zeta_{p}\right)}=H_{\mathbb{Q}\left(\zeta_{p}\right)}[9$, Proposition 3.4].

We have $F:=F_{\rho} \subseteq L^{\mathfrak{p}}$, where $\mathfrak{p}$ is the unique prime of $L$ above $p$. Suppose that the ramification degree $e=e(F / L)$ of $\mathfrak{p}$ in $F$ is 3 or 9 . Then by Lemma 2, the ramification in $F / L$ must be obtained by adjoining the unique degree $3(e=3)$ or $9(e=9)$ subfield of $\mathbb{Q}\left(\zeta_{p}\right)$ to $L$. It follows that $\left|P_{1}^{\prime}\right|=\left[F: F \cap \mathbb{Q}^{a b}\right]=3(e=3)$ or $1(e=9)$. This is a contradiction since $P_{1}^{\prime}=C_{3} \times C_{3}$. Therefore, $F / L$ is unramified and $F \subseteq H_{L}$.

We have the following lemma of Tate:

Lemma 3. (Tate [17, Theorem 5]) For each prime $p$, let $I_{p}$ be the inertia group of $p$ in $\mathrm{G}_{\mathbb{Q}_{p}}$, which we view as a fixed subgroup of $\mathrm{G}_{\mathbb{Q}}$. Let $\tilde{\rho}: \mathrm{G}_{\mathbb{Q}} \rightarrow \mathrm{PGL}_{n}(\mathbb{C})$ be a projective 
representation. Suppose that for each $p$, there exists a lift $\rho_{p}: \mathrm{G}_{\mathbb{Q}_{p}} \rightarrow \mathrm{GL}_{n}(\mathbb{C})$ of $\left.\tilde{\rho}\right|_{\mathrm{G}_{\mathbb{Q}_{p}}}$ with $\left.\rho_{p}\right|_{I_{p}}$ trivial for all but finitely many $p$. Then there exists a unique lift $\rho: \mathrm{G}_{\mathbb{Q}} \rightarrow \mathrm{GL}_{n}(\mathbb{C})$ of $\tilde{\rho}$ such that $\left.\rho\right|_{I_{p}}=\left.\rho_{p}\right|_{I_{p}}$ for all $p$.

Returning to our notation before Lemma 3, since $F_{\rho} / \mathbb{Q}$ is unramified outside $p$, for all primes $l \neq p,\left.\tilde{\rho}\right|_{\mathbb{G}_{\mathbb{Q}_{l}}}$ factors through a cyclic extension of $\mathbb{Q}_{l}$. The Schur multiplier $H_{2}(G, \mathbb{Z})$ of a cyclic group $G$ is trivial, so any projective representation of $G$ lifts to an ordinary representation of $G$ (see [10, Chapter 20], in particular Theorem 20.8, part c). We may thus lift $\left.\tilde{\rho}\right|_{\mathbb{G}_{\mathbb{Q}_{l}}}$ to a map $\rho_{l}: \mathrm{G}_{\mathbb{Q}_{l}} \rightarrow \mathrm{GL}_{3}(\mathbb{C})$ such that the fixed field of the kernel of $\left.\tilde{\rho}\right|_{\mathrm{G}_{\mathbb{Q}_{l}}}$ is the same as the fixed field of the kernel of $\rho_{l}$; in particular, both fixed fields are unramified at $l$.

Since $F_{\rho} / L$ is unramified, $F_{\rho} \mathbb{Q}\left(\zeta_{p}\right) / \mathbb{Q}\left(\zeta_{p}\right)$ is unramified. By class field theory, the principal ideal $\left(1-\zeta_{p}\right)$ splits completely in $F_{\rho} \mathbb{Q}\left(\zeta_{p}\right)$. It follows that the ideal $\mathfrak{p}$ of $L$ splits completely in $F_{\rho}$. Thus the kernel of the fixed field of $\left.\tilde{\rho}\right|_{\mathbb{Q}_{\mathbb{Q}_{p}}}$ is a totally tamely ramified extension of $\mathbb{Q}_{p}$, so cyclic. We may therefore lift $\left.\tilde{\rho}\right|_{\mathrm{G}_{\mathbb{Q}_{p}}}$ to a map $\rho_{p}$ such that the kernel of the fixed field of $\rho_{p}$ is the same as the kernel of the fixed field of $\left.\tilde{\rho}\right|_{\mathrm{G}_{\mathbb{Q}_{p}}}$.

Putting this local data together, Lemma 3 now tells us that there exists a lift $\rho$ of $\tilde{\rho}$ such that the ramification in $F_{\rho} / \mathbb{Q}$ is the same as the ramification in $E_{\rho} / \mathbb{Q}$ (i.e., $E_{\rho}$ is unramified at all primes $l \neq p$ and the ramification degree at $p$ in $E_{\rho} / \mathbb{Q}$ is the same as the ramification degree at $p$ in $\left.F_{\rho} / \mathbb{Q}\right)$.

We will need the following theorem:

Theorem 3. (Fröhlich [7, page 31; page 43, Corollary 2]) Let $L / K$ be Galois and let $N$ be a central extension of $L / K$. Then there is a surjective map

$$
H_{2}(\mathrm{G}(L / K), \mathbb{Z}) \rightarrow \mathrm{G}\left(N / L\left(N \cap K^{a b}\right)\right),
$$

and there exists a central extension $N^{\prime}$ of $L / K$ such that

$$
H_{2}(\mathrm{G}(L / K), \mathbb{Z}) \rightarrow \mathrm{G}\left(N^{\prime} / L\left(N^{\prime} \cap K^{a b}\right)\right)
$$

is an isomorphism. Moreover, if $K=\mathbb{Q}$, one can take $N^{\prime}$ to be ramified at the same rational primes that $L$ is ramified at.

Remark 2. The Schur multiplier $H_{2}(G, \mathbb{Z})$ of a group $G$ is intimately related to the study of central extensions and projective representations of $G$. See, for example, [10, Chapter 20].

Lemma 4. For a prime $p \equiv 1(\bmod 4)$, let $L \subseteq \mathbb{Q}\left(\zeta_{p}\right)$ be the unique field with $[L: \mathbb{Q}]=4$. Suppose that $L$ admits an unramified $C_{3} \times C_{3}$ extension $F$. Then $F / \mathbb{Q}$ has a unique degree three unramified central extension.

Proof. We first show the existence of such an extension. Let $\tilde{\rho}$ be a projective representation of $\mathrm{G}(F / \mathbb{Q})$ with image isomorphic to $P_{1}$. Let $\rho$ be a lift of $\tilde{\rho}$ such that the image of $\rho \mid I_{p}$ has order 4, the existence of which is guaranteed by Lemma 3. Let $N$ be the fixed field of ker $\rho$. Since the ramification degree of $p$ in $L$ is $4, N / L$ is unramified (recall we have assumed that $F / L$ is unramified) and $N \cap F \mathbb{Q}^{a b}=F$. By Theorem [3, we must have $\left[N: N \cap F \mathbb{Q}^{a b}\right]=$ 
$[N: F] \leq 3$. We cannot have $N=F$ because the group $P_{1}$ does not have any irreducible three-dimensional representations, so $[N: F]=3$. Thus $N$ gives the desired extension.

Now for uniqueness. Suppose that $N_{1}$ and $N_{2}$ are distinct non-trivial unramified extensions of $F / \mathbb{Q}$. We have $H_{2}(\mathrm{G}(F / \mathbb{Q}), \mathbb{Z})=C_{3}$ and $F\left(N_{i} \cap \mathbb{Q}^{a b}\right) \subseteq N_{i} \cap F \mathbb{Q}^{a b}$, so it follows from Theorem 3 that $C_{3} \rightarrow \mathrm{G}\left(N_{i} / N_{i} \cap F \mathbb{Q}^{a b}\right), i=1,2$. Since $N_{i} / F$ and $F / L$ are unramified, we have $N_{i} \cap F \mathbb{Q}^{a b}=F$. Suppose $N_{1} \neq N_{2}$. Then $N_{1} N_{2} / F$ is an unramified central extension of $F / \mathbb{Q}$ with $N_{1} N_{2} \cap F \mathbb{Q}^{a b}=F$ and $\left[N_{1} N_{2}: F\right]=9$, contradicting Theorem 3 ,

Lemma 5. Let $F / \mathbb{Q}$ be a finite Galois extension with group $G$, and suppose $H_{2}(G, \mathbb{Z}) \cong C_{3}$. For a fixed number field $M \subset \mathbb{Q}\left(\zeta_{p^{\infty}}\right), F M / \mathbb{Q}$ admits a maximum of three distinct non-trivial central extensions $E_{i}$ with $E_{i} \cap \mathbb{Q}^{a b}=M$ and $E_{i} / \mathbb{Q}$ unramified outside $p \infty$.

Proof. Let $E_{i}$ be fields as in the statement of the lemma. By Theorem 3, for each $i$, we have $\left[E_{i}: F M\right]=3$. The compositum $\prod E_{i}$ is a central extension of $F M / \mathbb{Q}$. By Theorem 3 , there exists $M^{\prime} \subset \mathbb{Q}\left(\zeta_{p^{\infty}}\right)$ containing $M$ such that $\left|\mathrm{G}\left(\prod E_{i} / F M^{\prime}\right)\right| \leq 3$. This means $\prod E_{i} / F M$ has rank at most two, i.e., its Galois group is $C_{3}$ or $C_{3} \times C_{3}$. Such a field extension has at most (in the $C_{3} \times C_{3}$ case) four strictly intermediate extensions- three $E_{i}$ 's and $F M^{\prime}$, (and we must have $\left.\left[M^{\prime}: M\right]=3\right)$.

We now come to the main result of this section.

Proposition 1. Let $\rho: \mathrm{G}_{\mathbb{Q}} \rightarrow \mathrm{GL}_{3}(\mathbb{C})$ be an Artin representation unramified outside $p \infty$ with projective image isomorphic to $P_{1}$. Let $F$ be the fixed field of $\operatorname{ker} \pi \circ \rho$. Let $\mathbb{Q}\left(\zeta_{p, 3}\right)$ denote the maximal subfield of $\mathbb{Q}\left(\zeta_{p}\right)$ of 3-power degree over $L$, and let $\mathbb{Q}\left(\zeta_{p, 3^{\prime}}\right)$ be the composite of all subfields of $\mathbb{Q}\left(\zeta_{p^{\infty}}\right)$ with degree prime-to-3 over $L$. Then the fixed field of ker $\rho$ either

i) lies between $N$ and $N \mathbb{Q}\left(\zeta_{p, 3^{\prime}}\right)$, where $N$ is the unique non-trivial unramified central extension of $F / \mathbb{Q}$, and $[N: F]=3$, or

ii) lies between $E_{3}$ and $E_{3} \mathbb{Q}\left(\zeta_{p, 3^{\prime}}\right)$, where $E_{3} \nsubseteq F \mathbb{Q}\left(\zeta_{p, 3}\right)$ is one of two possible degree 3 ramified extensions of $F M_{3}$ that is central over $F / \mathbb{Q}$, where $M_{3}$ is a subfield of $\mathbb{Q}\left(\zeta_{p, 3}\right)$.

Proof. The existence and uniqueness of $N$ is given by Lemma 4 . For the entirety of the proof, we consider representations $\rho$ for which $F_{\rho}=F$, with $F$ fixed. Let $E$ be a candidate for the fixed field of $\operatorname{ker} \rho$. By Theorem 3 , there exists $M \subset \mathbb{Q}\left(\zeta_{p^{\infty}}\right)$ such that $[E: F M] \leq 3$, and $E \cap \mathbb{Q}^{a b}=M$. We claim that $E \neq F M$. If $E$ were contained in $F M$ with $[E: F]=2^{k} m, m$ odd, then we would have $\mathrm{G}(E / \mathbb{Q}) \cong\left(C_{3} \times C_{3}\right) \rtimes\left(C_{2^{k}} \times C_{m}\right)$, with $C_{2^{k}} \times C_{m}$ acting on $C_{3} \times C_{3}$ through its $C_{4}$ quotient. Thus $C_{3} \times C_{3} \times C_{m}$ is an abelian normal subgroup of $\mathrm{G}(E / \mathbb{Q})$ of index $2^{k}$. By [2, Corollary 53.18], the degree of every irreducible representation of $\mathrm{G}(E / \mathbb{Q})$ divides $2^{k}$. In particular, $\mathrm{G}(E / \mathbb{Q})$ has no irreducible three-dimensional representations, a contradiction.

The proof will be complete upon establishing the following two claims.

Claim I: If $E / F M$ is unramified, then we are in Case i) of the proposition. 
Claim II: If $E / F M$ is ramified, then we are in Case ii) of the proposition.

Proof of Claim I. We are assuming that $E / F M$ is unramified. Being a composite of two central extensions of $F, N M$ is a central extension of $F / \mathbb{Q}$ as well (see Figure $\mathbb{1}$ ).
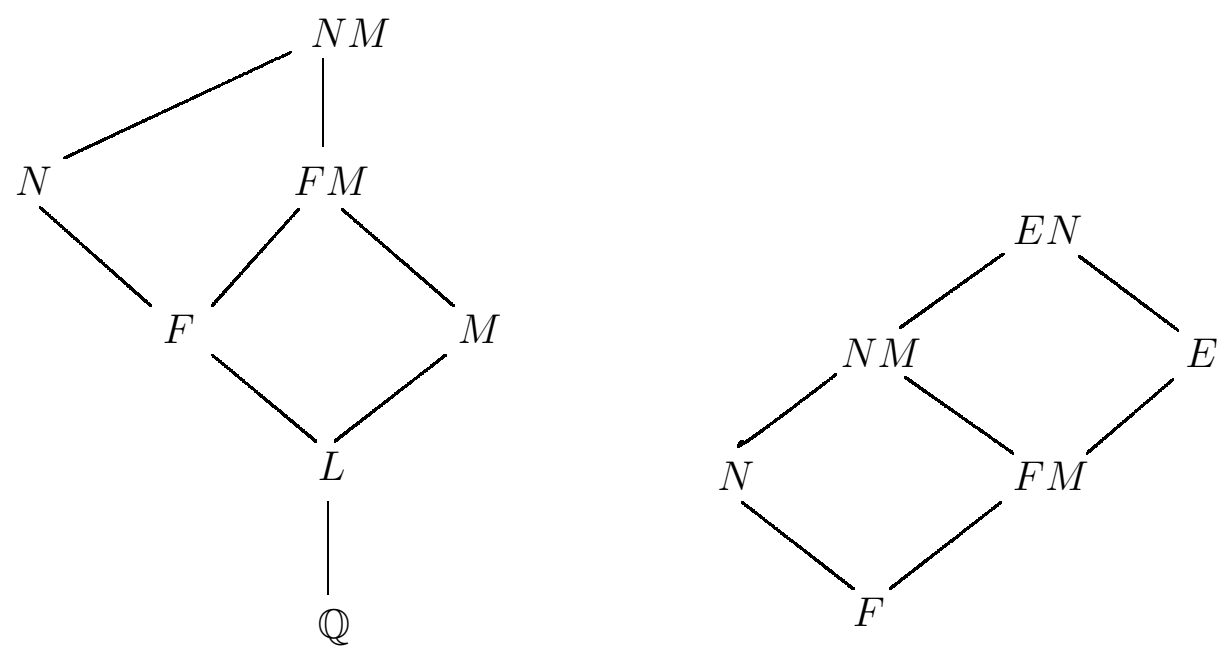

Figure 1: Field diagrams for proof of Proposition 1.

If $E \neq N M$, then $E N$ is an unramified central extension of $F M$. Since $F / L$ is unramified, if $E N / F M$ is unramified, then $E N \cap F M \mathbb{Q}^{a b}=F M$ with $[E N: F M]=9$, which contradicts Theorem 3, So $E=N M$, and we have only to show that $M \subseteq \mathbb{Q}\left(\zeta_{p, 3^{\prime}}\right)$. Suppose that $3 \mid[M: \mathbb{Q}]$. Then $N M / F$, having two intermediate subfields of degree 3 over $F$, is not cyclic. Thus we must have $M \subseteq \mathbb{Q}\left(\zeta_{p, 3^{\prime}}\right)$.

Proof of Claim II. Let $M_{3}=M \cap \mathbb{Q}\left(\zeta_{p, 3}\right)$ and $M_{3^{\prime}}=M \cap \mathbb{Q}\left(\zeta_{p, 3^{\prime}}\right)$, so $M=M_{3} M_{3^{\prime}}$. Since $E / F M_{3}$ is cyclic (recall that $E / F$ is cyclic) and $\left[E: F M_{3}\right]$ is divisible by 3 but not by $3^{2}$, there exists a degree 3 extension $E_{3}$ of $F M_{3}$ such that $E=E_{3} M$. Note that $E_{3} M=E_{3} M_{3^{\prime}}$. An elementary ramification argument shows that $E / F M_{3}$, and thus $E_{3} / F M_{3}$, is totally ramified. Define

$\Sigma_{M_{3}}=\left\{\right.$ Central extensions $J$ of $F / \mathbb{Q}$ containing $M_{3}: J / F M_{3}$ is ramified at $p$ and only at $p$,

$$
\left.\left[J: F M_{3}\right]=3 \text {, and } J \cap \mathbb{Q}^{a b}=M_{3}\right\} .
$$

We are assuming $E_{3} / F M_{3}$ is ramified, so $E_{3} \in \Sigma_{M_{3}}$. From Lemma吕, we have $\# \Sigma_{M_{3}} \leq 3$. To complete the proof of Claim II, we need to show that $\# \Sigma_{M_{3}} \leq 2$. From the proof of Lemma 5. the compositum $J^{\prime}$ of all elements of $\Sigma_{M_{3}}$ is contained in a $C_{3} \times C_{3}$ extension of $F M_{3}$. We may assume $\# \Sigma_{M_{3}}=3$, and thus that $J^{\prime}$ is equal to a $C_{3} \times C_{3}$ extension of $F M_{3}$. The proof of Lemma 5 also tells us that $F M^{\prime} \subset J^{\prime}$, where $M^{\prime} \subset \mathbb{Q}\left(\zeta_{p^{\infty}}\right)$ and $\left[M^{\prime}: M_{3}\right]=3$. Since $F / L$ is unramified, $F M_{3} / M_{3}$ is unramified as well, from which it follows that $F M^{\prime} / F M_{3}$ is ramified, as $M^{\prime} / \mathbb{Q}$ is totally ramified. If $\# \Sigma_{M_{3}}=3$, then all four intermediate subfields of 
$J^{\prime} / F M_{3}$ are ramified over $F M_{3}$, and thus $J^{\prime} / F M_{3}$ is totally ramified. This is a contradiction, however, since the corresponding extensions of local fields at primes above $p$ would be totally tamely ramified but not cyclic.

The next goal is to count the number of representations $\mathrm{G}_{\mathbb{Q}} \rightarrow \mathrm{GL}_{3}(\mathbb{C})$ with projective image isomorphic to $P_{1}$ and given prime power conductor. We must have $p \equiv 1(\bmod 4)$, and we will also assume (as is necessary for such a representation to exist), that the 3-rank $k$ of the class group $\mathrm{Cl}(L)$ of $L$ is at least 2. Then $L$ admits $r_{3,2}(k)$ unramified extensions $F$ with $\mathrm{G}(F / L)=C_{3} \times C_{3}$.

Let $J=\mathrm{G}(N / \mathbb{Q})$, where $N$ is as in Case i) of Proposition 11. Since the 3-Sylow subgroup $\mathrm{G}(N / L):=A$ of $J$ is normal in $J$, the group $J$ splits as $A \rtimes C_{4}$. If $A$ is abelian, then $J$ is metabelian (i.e., the second commutator group of $G$ is trivial), which gives a contradiction since metabelian groups are monomial [2, Theorem 52.2], and $\rho$ is primitive. Suppose that $A$ is isomorphic to the non-trivial semi-direct product $C_{9} \rtimes C_{3}$. A generator of $C_{4}$ acts on $J / Z(J)$ as an automorphism of order 4 and thus must act on $A$ as an automorphism of order a multiple of 4 . But an exercise in group theory shows that $\left|\operatorname{Aut}\left(C_{9} \rtimes C_{3}\right)\right|=54$, so no such automorphism exists. Thus $A$ must be the other non-abelian group of order $27,\left(C_{3} \times\right.$ $\left.C_{3}\right) \rtimes C_{3}$. A computer calculation [8] shows the group $J$ has 8 irreducible three-dimensional representations. Let $S$ denote the set of these representations. As an example, we determine the conductors of these representations- that is, the conductors of the representations of $\mathrm{G}_{\mathbb{Q}}$ for which the fixed field of the kernel is $N$. Recall the definition of the Artin conductor $N(\rho)$ of a representation $\rho$ from Section [1. We have $J_{0}=C_{4}, J_{i}=0, i \geq 1$. Using [8], one finds that for 2 of the 8 aforementioned representations, $\operatorname{dim} V^{J_{0}}=0$, and for the other 6 , $\operatorname{dim} V^{J_{0}}=1$, where $V \cong \mathbb{C}^{3}$ is the representation space. So we get two representations with conductor $p^{3}$ and 6 with conductor $p^{2}$.

Suppose now that we are in Case i) of Proposition 1, so the fixed field of the kernel of a representation $\rho$ is of the form $N M$, with $M \subset \mathbb{Q}\left(\zeta_{p, 3^{\prime}}\right)$. The irreducible three-dimensional representations of $\mathrm{G}(N M / \mathbb{Q})$ are given by $\psi \otimes \chi$, where $\psi$ is an element of $S$ and $\chi$ is a character of $\mathrm{G}(M / L)$, of which there are $[M: L]$ (by abuse of notation, we are allowing $\rho$ and $\chi$ to also denote their respective lifted representations to the group $\mathrm{G}(N M / \mathbb{Q})$; furthermore, we are identifying characters of $G(M / L)$ with characters of $G(M / \mathbb{Q})$ modulo characters of $G(L / \mathbb{Q})$ ). To see this, note that the sum of the squares of the degrees of the irreducible representations of $\mathrm{G}(N M / \mathbb{Q})$ is equal to the order of the group. Consider the irreducible representations of $\mathrm{G}(N M / \mathbb{Q})$ the form $\psi \otimes \chi$, where $\psi$ ranges over all irreducible representations of $\mathrm{G}(N / \mathbb{Q})$ and $\chi$ ranges over all irreducible representations of $\mathrm{G}(M / L)$. The sum of the squares of the degrees of these representations is the order of $\mathrm{G}(N M / \mathbb{Q})$. If we can show that these representations are distinct as $\psi$ and $\chi$ vary, then it will follow that these are all the irreducible representations of $\mathrm{G}(N M / \mathbb{Q})$. This amounts to showing that for distinct representations $\psi$ and $\psi^{\prime}$ of $G(N / \mathbb{Q})$ we cannot have $\psi \otimes \chi \cong \psi^{\prime}$. One can see this by considering the restrictions of both representations to $G(N M / N)$.

Thus we find that the number of irreducible representations of $\rho$ of dimension three with ker $\rho$ having fixed field $N M$ is $8[M: L]$.

Continuing with Case i) of Proposition 1, we now fix a $C_{3} \times C_{3}$ extension $F$ of $L$ and 
count the number of $\rho$ for which $N(\rho)=p^{m}$ and $F_{\rho}=F$. Write $[M: \mathbb{Q}]=4 c p^{n}$, with $c \mid \frac{p-1}{4}$, so

$[M: L]=p^{n} c$. Let $G=\mathrm{G}(N M / \mathbb{Q})$, and let $G_{i}$, with order $g_{i}$, denote the lower ramification groups of $N M / \mathbb{Q}$. (Technically, we mean the lower ramification groups of the extension $N M_{\mathfrak{p}} / \mathbb{Q}_{p}$ of local fields where $\mathfrak{p}$ is a prime of $N M$ lying above $p$. Since $N M / \mathbb{Q}$ is Galois and ramified only at $p$, there is no ambiguity created by this language, and we use it henceforth when we have a Galois extension of $\mathbb{Q}$ ramified at a single rational prime). As soon as $M \nsubseteq N$, we get non-trivial central elements in $G_{i}$ whenever $G_{i} \neq 1$. Such elements act by scalars and thus do not fix any non-zero subspace of $V$. So for such $\rho, v_{p}(N(\rho))=\sum_{i \geq 0, g_{i} \neq 1} \frac{g_{i}}{g_{0}} \cdot 3$. We must compute the $g_{i}$. We know $g_{0}=4 c p^{n}, g_{1}=p^{n}$.

Lemma 6. Let $E / \mathbb{Q}$ be a Galois extension unramified outside $p \infty$ and tamely ramified at $p$. Let $M$ be subfield of $\mathbb{Q}\left(\zeta_{p^{n}}\right)$ that is not contained in $\mathbb{Q}\left(\zeta_{p^{n-1}}\right)$. Then for $i \geq 1$, the $i$ th lower ramification groups of $\mathrm{G}(E M / \mathbb{Q})$ have the same order as the respective lower ramification groups of $\mathbb{Q}\left(\zeta_{p^{n}}\right) / \mathbb{Q}$.

Proof. Let $G=\mathrm{G}(E M / \mathbb{Q}), G^{\prime}=\mathrm{G}\left(\mathbb{Q}\left(\zeta_{p^{n}}\right) / \mathbb{Q}\right)$, with upper ramification groups $G^{i}$ and $G^{\prime i}$, respectively, and let $H=\mathrm{G}(E M / M), H^{\prime}=\mathrm{G}\left(\mathbb{Q}\left(\zeta_{p^{n}}\right) / M\right)$. Since $G^{i}$ is a $p$-group, for all $i>0$,

$$
\begin{aligned}
& (G / H)^{i}=G^{i} H / H=G^{i} / G^{i} \cap H=G^{i} \text { and } \\
& \left(G^{\prime} / H^{\prime}\right)^{i}=G^{\prime i} H^{\prime} / H^{\prime}=G^{\prime i} / G^{\prime i} \cap H^{\prime}=G^{\prime i},
\end{aligned}
$$

where the first equality in both lines is a property of upper ramification groups (see [19, Chapter IV] for details about the upper and lower ramification groups). The order of the lower ramification groups is determined by the order of the upper ramification groups. Since $(G / H)^{i}=\left(G^{\prime} / H^{\prime}\right)^{i}$, (5) and (6) imply that $\left|G_{i}\right|=\left|G_{i}^{\prime}\right|$ for all $i \geq 1$.

Let $G^{\prime}:=\mathrm{G}\left(\mathbb{Q}\left(\zeta_{p^{n+1}}\right) / \mathbb{Q}\right)$. As shown in [19, Chapter IV.4], for any $0 \leq k \leq n$,

$$
\left|G_{i}^{\prime}\right|=p^{k} \quad \text { for } \quad p^{n-k} \leq i \leq p^{n-k+1}-1, \quad i \in \mathbb{Z} .
$$

By Lemma 6, $\left|G_{i}\right|=\left|G_{i}^{\prime}\right|$ for all $i \geq 1$. So $\left|G_{i}\right|=p^{k}$ for $p^{n-k} \leq i \leq p^{n-k+1}-1$ and $0 \leq k \leq n$, and $\left|G_{i}\right|=1$ for $i \geq p^{n}$. Thus if $\operatorname{ker} \rho$ has fixed field $N M$,

$$
v_{p}(N(\rho))=3\left(1+\frac{p-1}{4 c}+\frac{p(p-1)}{4 c p}+\cdots+\frac{p^{n-1}(p-1)}{4 c p^{n-1}}\right)=3+3 n \frac{p-1}{4 c},
$$

and there are $8 p^{n} c$ representations with kernel fixing $N M$.

We are now in a position to estimate the number $a_{m, i}$ of $\rho$ for which we are in Case i) of Proposition 1 and for which $N(\rho)=p^{m}$ and $F_{\rho}=F$. If $m \equiv 1$ or $2(\bmod 3)$, then $a_{m, i}=0$. Suppose $m \equiv 0(\bmod 3)$. Let $d=\operatorname{gcd}\left(\frac{p-1}{4}, k\right)$, where $m=3 k+3$. Then

$$
a_{m, i}=8 p^{k} \frac{p-1}{4}+\cdots+8 p^{\frac{k}{d}} \frac{p-1}{4 d}=8 \sum_{j \mid d} p^{\frac{k}{j}} \frac{p-1}{4 j} .
$$


The first term in the sum, for example, corresponds to the case $c=\frac{p-1}{4}, n=k$, in which case there exist $8 p^{k} \frac{p-1}{4}$ representations $\rho$ with $v_{p}(N(\rho))=3+3 n \frac{p-1}{4 c}=3+3 k$.

For the total number $A_{m, F, i}$ of representations $\rho$ in Case i) of Proposition 1 with $F_{\rho}=F$ and $N(\rho) \mid p^{m}$, we have

$$
A_{m, F, i} \sim \sum_{0 \leq k \leq \frac{m-3}{3}} 2 p^{k+1} \sim 2 p^{\frac{m}{3}},
$$

where we are viewing $A_{m, F, i}$ as a function of $p$.

Suppose now that we are in Case ii) of Proposition 1. We can write the fixed field of ker $\rho$ as $E_{3} M_{3^{\prime}}$, with $M_{3^{\prime}} \subset \mathbb{Q}\left(\zeta_{p, 3^{\prime}}\right)$ and $E_{3}$ a degree 3 ramified extension of $F M_{3}$ not contained in $F \mathbb{Q}\left(\zeta_{p, 3}\right)$, with $M_{3} \subseteq \mathbb{Q}\left(\zeta_{p, 3}\right)$. Let $\left[M_{3}: L\right]=3^{a}$. The group $\mathrm{G}\left(E_{3} / \mathbb{Q}\right)$ splits as $B \rtimes C_{4}$, where $B=\mathrm{G}\left(E_{3} / L\right)$ is a 3 -group.

Claim: $B \cong\left(C_{3^{a+1}} \times C_{3}\right) \rtimes C_{3}$, where $Z(B)$ is the first $C_{3^{a+1}}$ factor, which is also the center of $\mathrm{G}\left(E_{3} / \mathbb{Q}\right)$ (a generator of the last $C_{3}$ factor acts on $C_{3^{a+1}} \times C_{3}$ by fixing the $C_{3^{a+1}}$ factor and mapping $\left.(0,1) \mapsto\left(3^{a}, 1\right)\right)$.

Proof of Claim. The same reasoning that showed $A \cong\left(C_{3} \times C_{3}\right) \rtimes C_{3}$ in the discussion immediately following the proof of Proposition 1 shows that $\mathrm{G}\left(E_{3} / M_{3}\right) \cong\left(C_{3} \times C_{3}\right) \rtimes C_{3}$. Since $E_{3} / F$ is cyclic, it follows that if $F^{\prime}$ is a field strictly between $L$ and $F$, then $\mathrm{G}\left(E_{3} / F^{\prime}\right)$ has rank two (see Figure 2). The group $\mathrm{G}\left(E_{3} / F^{\prime}\right)$ is abelian since its center has index at most three. Thus $\mathrm{G}\left(E_{3} / F^{\prime}\right) \cong C_{3^{a+1}} \times C_{3}$, and it is normal in $B$ since $F^{\prime} / L$ is Galois. Since $\mathrm{G}\left(E_{3} / M_{3}\right) \cong\left(C_{3} \times C_{3}\right) \rtimes C_{3}$, it has a subgroup $C$ of order three not contained in $\mathrm{G}\left(E_{3} / F^{\prime}\right)$, and thus $B$ splits as $\mathrm{G}\left(E_{3} / F^{\prime}\right) \rtimes C$, as claimed.

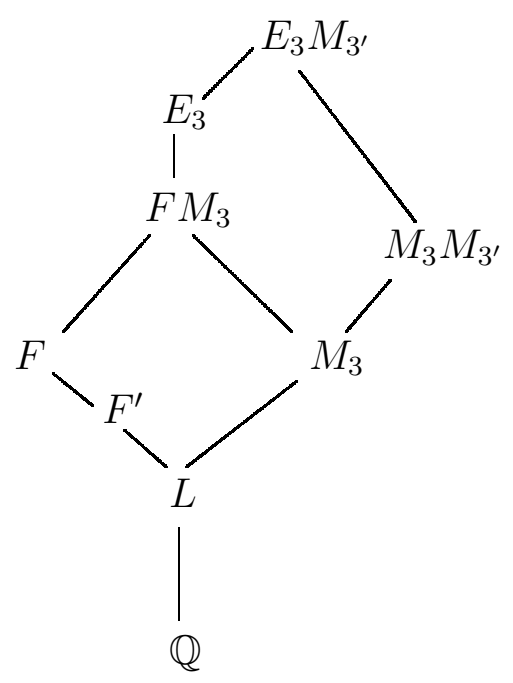

Figure 2:

We would now like to count the number of irreducible representations of $G\left(E_{3} / \mathbb{Q}\right) \cong$ $B \rtimes C_{4}$. While counting representations from Case i) of Proposition 1, we saw that 
$\left(\left(C_{3} \times C_{3}\right) \rtimes C_{3}\right) \rtimes C_{4} \cong\left(\left(3^{a} C_{3^{a+1}} \times C_{3}\right) \rtimes C_{3}\right) \rtimes C_{4}:=B^{\prime} \rtimes C_{4}$ has 8 irreducible threedimensional representations. If $\psi$ is an irreducible representation of $B^{\prime} \rtimes C_{4}$, and $\psi$ maps the generator $3^{a} \in 3^{a} \mathbb{Z} / 3^{a+1} \mathbb{Z}$ of the $3^{a} C_{3^{a+1}}$ factor to the scalar $\alpha \in \mathrm{GL}_{3}(\mathbb{C})$, then mapping the generator $1 \in \mathbb{Z} / 3^{a+1} \mathbb{Z}$ of the $C_{3^{a+1}}$ factor to any of $3^{a}$ th roots of $\alpha$ gives a well defined irreducible representation of $B \rtimes C_{4}$, and distinct choices of $3^{a}$ th roots of $\alpha$ give distinct representations. Using the fact that the sum of the squares of the degrees of the irreducible representations is the order of the group, we see that we obtain all irreducible representations of $B \rtimes C_{4}$ in this way. It follows that the number of irreducible three-dimensional representations of $\mathrm{G}\left(E_{3} / \mathbb{Q}\right)$ is $8 \cdot 3^{a}$. Recall that in Case ii), the fixed field of the kernel of our given representation $\rho$ is of the form $E_{3} M_{3^{\prime}}$, where $M_{3^{\prime}} \subseteq \mathbb{Q}\left(\zeta_{p, 3^{\prime}}\right)$. The number of irreducible three-dimensional representations of $\mathrm{G}\left(E_{3} M_{3^{\prime}} / \mathbb{Q}\right)$ is thus $8 \cdot 3^{a} \cdot\left[M_{3} M_{3^{\prime}}: M_{3}\right]$.

Write $\left[E_{3} M_{3^{\prime}}: \mathbb{Q}\right]=108 p^{n} c$, with $(c, p)=1$. Then our number $8 \cdot 3^{a} \cdot\left[M_{3} M_{3^{\prime}}: M_{3}\right]$ from above is equal to $8 p^{n} c$. Let $G_{i}$, with order $g_{i}$, be the lower ramification groups of $\mathrm{G}\left(E_{3} M_{3^{\prime}} / \mathbb{Q}\right)$. For all $i \geq 0, G_{i}$ has non-trivial central elements, so $\operatorname{dim} V / V^{G_{i}}=3$ whenever $g_{i} \neq 1$. So again we obtain

$$
v_{p}(N(\rho))=\sum_{i \geq 0, g_{i} \neq 1} \frac{g_{i}}{g_{0}} \cdot 3
$$

and we have to compute the $g_{i}$. We have $g_{0}=12 p^{n} c$. It follows from Lemma 6 that the $g_{i}$ for Case ii) are the same as those for Case i). We are thus able to compute, analogously to Case i),

$$
v_{p}(N(\rho))=3+3 n \frac{p-1}{12 c} .
$$

In the same way that we bounded $a_{m, i}$, we can now bound the number $a_{m, i i}$ of $\rho$ for which we are in Case ii) of Proposition 1 and for which $N(\rho)=p^{m}$ and $F_{\rho}=F$. By (7), $a_{m, i i}=0$ unless $m \equiv 0(\bmod 3)$, so write $a_{m, i i}=3 k+3$. A similar series of computations to those in Case i) gives

$$
a_{m, i i}=8 p^{k} \frac{p-1}{4}+\cdots+8 p^{\frac{k}{d}} \frac{p-1}{12 d}=8 \sum_{j \mid d} p^{\frac{k}{j}} \frac{p-1}{12 j}, \quad d=\operatorname{gcd}\left(\frac{p-1}{4}, k\right) .
$$

When we are in Case ii), for a fixed $M_{3}$ and $F$, there are two possible degree 3 extensions of $\mathrm{FM}_{3}$ that are candidates for the kernel of the fixed field of the representation $\rho$, so we obtain

$$
A_{m, F, i i} \sim \sum_{0 \leq k \leq \frac{m-3}{3}} \frac{4}{3} p^{k+1} \sim \frac{4}{3} p^{\frac{m}{3}} .
$$

To bound the total number $B_{m}$ of primitive representations $\rho$ with $N(\rho) \mid p^{m}$ and $\operatorname{Im}(\pi \circ \rho)=P_{1}$, we need to multiply $A_{m, F, i}+A_{m, F, i i}$ by the number of unramified $C_{3} \times C_{3}$ extensions of $L$, which, as previously discussed, is $r_{3,2}(k)$, where $k$ is the 3 -rank of $\mathrm{Cl}(L)$. From [15, page 95], the 3-part of $h_{L}$ is less than $\frac{22.2 p^{3}}{\pi^{4}}$, so $k \leq \log _{3}\left(\frac{22.2 p^{3}}{\pi^{4}}\right):=R$.

We introduce the following (non-standard) notation. Suppose that $A$ and $B$ are both positive, real-valued functions of $n$.

$$
\text { We write } A \lesssim B \text { to mean } \limsup _{n \rightarrow \infty} \frac{A(n)}{B(n)} \leq 1 \text {. }
$$


Using this notation, we obtain:

Theorem 4. Notation as above,

$$
B_{m} \lesssim\left(A_{m, F, i}+A_{m, F, i i}\right) r_{3,2}(R) \sim \frac{4928.4}{3^{5} \pi^{8}} p^{\frac{m}{3}+9}
$$

\subsection{Representations with projective image isomorphic to $P_{3}$}

Recall that $P_{3}:=\left(C_{3} \times C_{3}\right) \rtimes \mathrm{SL}_{2}\left(\mathbb{F}_{3}\right)$. Using the notation from the previous section, we look to count the number of $\rho$ with $\mathrm{G}\left(F_{\rho} / \mathbb{Q}\right) \cong P_{3}$ and given conductor. Our main tool will be the following proposition.

Proposition 2. Let $\rho: \mathrm{G}_{\mathbb{Q}} \rightarrow \mathrm{GL}_{3}(\mathbb{C})$ be an Artin representation unramified outside $p \infty$ with projective image isomorphic to $P_{3}$. Let $F$ be the fixed field of $\operatorname{ker} \pi \circ \rho$. If $M \subseteq \mathbb{Q}\left(\zeta_{p^{\infty}}\right)$, then there are at most three degree 3 central extensions of $F_{\rho} M / \mathbb{Q}$ ramified over $F_{\rho} M$ and at most one that is unramified over $F_{\rho} M$. There exists $M \subseteq \mathbb{Q}\left(\zeta_{p^{\infty}}\right)$ such that of the four possible degree 3 central extensions of $F_{\rho} M / \mathbb{Q}$, the fixed field of ker $\rho$ must be the unramified extension.

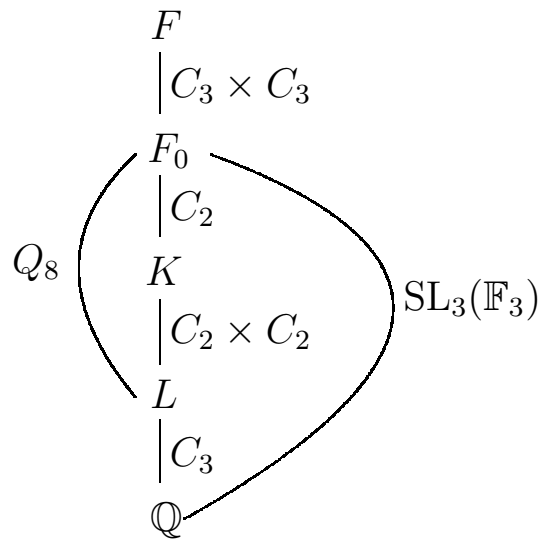

Figure 3: Field diagram for Section 4.2

Proof. We have $H_{2}\left(P_{3}, \mathbb{Z}\right) \cong C_{3}$. Let $E$ be a candidate for the fixed field of ker $\rho$ and let $M=E \cap \mathbb{Q}^{a b}$. By Lemma 5 and its proof, $F M / \mathbb{Q}$ admits a maximum of three distinct non-trivial central extensions $E_{i}$ with $E_{i} \cap \mathbb{Q}^{a b}=M$, each of which has degree 3 over $F M$, in addition to the degree 3 central extension $F M^{\prime}$ with $M^{\prime} \subseteq \mathbb{Q}\left(\zeta_{p^{\infty}}\right)$.

The group $\mathrm{G}\left(F M^{\prime} / \mathbb{Q}\right)$ has an abelian normal subgroup that is not cyclic, and it follows from [11, Corollary 6.13] that such a group has no faithful primitive representations, so $F M^{\prime}$ cannot be the fixed field of $\operatorname{ker} \rho$ (the same argument shows $F M^{\prime \prime}$ cannot be the fixed field of ker $\rho$ for any $\left.M^{\prime \prime} \subset \mathbb{Q}\left(\zeta_{p^{\infty}}\right)\right)$. Since we cannot have a totally ramified $C_{3} \times C_{3}$ extension of $F M$ that is Galois over $\mathbb{Q}$, we must have either at least one of the $E_{i}^{\prime} s$ be unramified or 
have $F M^{\prime} / F M$ unramified. If $F M^{\prime} / F M$ were unramified, ramification considerations force $e:=e\left(F / F_{0}\right)$ to be 3 or 9 , where $F_{0}$ is the fixed field of the $C_{3} \times C_{3}$ normal subgroup of $P_{3}$. This is impossible, however, since we would have $e(F / \mathbb{Q})=3 e$, so the extension of $\mathbb{Q}_{p}$ given by completing $F$ at a prime above $p$ would have a $C_{3 e}$ subgroup, and $P_{3}$ does not have such a subgroup. Thus one of the $E_{i}$ 's is unramified over $F M$. If two of the $E_{i}$ 's were unramified, the composite of these fields would give a $C_{3} \times C_{3}$ unramified central extension of $F M$ that contains $F M^{\prime}$, forcing $F M^{\prime} / F M$ to be unramified, which we just saw gives a contradiction.

Thus, $F M / \mathbb{Q}$ may have up to four central extensions of degree 3 , and at this point, three of them (all but $F M^{\prime}$ ) are possibilities for $E$. By Theorem 3 , $E$ is a degree 3 central extension of $F M / \mathbb{Q}$ for some $M \subset \mathbb{Q}\left(\zeta_{p^{\infty}}\right)$ since $C_{3}$ surjects onto $\mathrm{G}(E / F M)$. It remains to show that $E / F M$ must be unramified. Suppose that $E / F M$ is ramified. Write $[F M: F]=3^{a} b$ with $3 \nmid b$, and let $M_{3} \subset \mathbb{Q}\left(\zeta_{p^{\infty}}\right)$ be the field satisfying $\left[F M_{3}: F\right]=3^{a}$. The extension $F M_{3} / F$ is totally ramified since $F / F_{0}$ is unramified. We have $\mathrm{G}\left(F M_{3} / \mathbb{Q}\right) \cong\left(C_{3} \times C_{3}\right) \rtimes\left(Q_{8} \rtimes C_{3^{a+1}}\right)$ with the $C_{3^{a+1}}$ factor acting through its $C_{3}$ quotient. The 3-part of the inertia group at a prime above $p$ in $E$ is cyclic of order $3^{a+2}$. It follows that $\mathrm{G}(E / \mathbb{Q}) \cong\left(C_{3} \times C_{3}\right) \rtimes\left(Q_{8} \rtimes C_{3^{a+2}}\right)$ with the $C_{3^{a+2}}$ factor acting through its $C_{3}$ quotient. The left $\left(C_{3} \times C_{3}\right)$ factor is then an abelian normal subgroup of $\mathrm{G}(E / \mathbb{Q})$, which contradicts the primitivity of the representation.

Remark 3. Since $E / F$ is abelian (it is cyclic) and $E / F M$ is unramified, there exists a field $E_{0}$ between $E$ and $F$ such that $E_{0} / F$ is unramified of degree 3 and $E / E_{0}$ is totally ramified. Since $F M_{3} / F$ is totally ramified, $E_{0}$ cannot be contained in $F M_{3}$ (so $\left.E_{0} \nsubseteq F M\right)$, and thus we have $E=E_{0} M$. Since $E / F$ is cyclic, we also have that $[F M: F]$ is not divisible by 3 .

Remark 4. Unlike in the $P_{1}$ case, we may not use Lemma 3 here to guarantee the existence of an unramified degree 3 central extension of $F$ because the extension of $\mathbb{Q}_{p}$ given by completing at a prime above $p$ in $F$ is not necessary cyclic, so we are not assured of a lift $\rho_{p}: \mathrm{G}_{\mathbb{Q}_{p}} \rightarrow \mathrm{GL}_{3}(\mathbb{C})$ of $\left.\tilde{\rho}\right|_{\mathrm{G}_{\mathbb{Q}_{p}}}$ such that the fixed field of the kernel of $\rho_{p}$ is unramified over the fixed field of the kernel of $\left.\tilde{\rho}\right|_{\mathbb{G}_{\mathbb{Q}_{p}}}$.

Maintaining the notation from Figure 2, let $L=F^{a b}$ be the degree 3 extension of $\mathbb{Q}$ contained in $\mathbb{Q}\left(\zeta_{p}\right)$. The field $L$ then admits a $C_{2} \times C_{2}$ extension, which is unramified by Lemma 2. In the proof of Proposition 2 we saw that $F / F_{0}$ must be unramified, so $e(F / \mathbb{Q})=3$ or 6 .

Continuing in the manner of the $P_{1}$ case, we now bound, for a fixed $F / \mathbb{Q}$ with $G(F / \mathbb{Q}) \cong$ $P_{3}$, the number of $\rho$ for which $N(\rho)=p^{m}$ and $F_{\rho}=F$. Let $E=E_{\rho}$, so $E$ is of the form $E_{0} M$ for some $M \subseteq \mathbb{Q}\left(\zeta_{p^{\infty}}\right)$, where $E_{0}$ is as in Remark 3, Given $M$, by Proposition 2 there is one possible choice for $E$. Write $[M: L]=p^{n} c$, with $c \mid \frac{p-1}{3}$. We have $e(E / \mathbb{Q})=3 p^{n} c x$, where $x=1$ or 2 . Using Lemma 6 to compute analogously to the $P_{1}$ case, we find

$$
v_{p}\left(N_{\rho}\right)=3+3 n \frac{p-1}{3 c x} .
$$

An exhaustive search using [8] shows that there are 3 non-split central extensions of $P_{3}$ of order $3\left|P_{3}\right|=648$, and using [8], we can check that each of them has 7 irreducible three-dimensional representations. 
Remark 5. We are considering central extensions of $P_{3}$ by $C_{3}$ up to group isomorphism, not up to isomorphism of central extensions, which are classified by $\mathrm{H}^{2}\left(P_{3}, C_{3}\right)$.

The three-dimensional irreducible representations of $\mathrm{G}(E / \mathbb{Q})$ are all the $\psi \otimes \chi$, as $\psi$ runs over the three-dimensional irreducible representations of $\mathrm{G}\left(E_{0} / \mathbb{Q}\right)$ and $\chi$ runs over all characters of $\mathrm{G}\left(E / E_{0}\right) \cong \mathrm{G}(M / L)$. Thus $\mathrm{G}(E / \mathbb{Q})$ has $7 p^{n} c$ irreducible three-dimensional representations. We estimate the number $a_{m, F}$ of representations $\rho$ with $N(\rho)=p^{m}$ and $F_{\rho}=F$. We must have $3 \mid m$ so write $m=3 k+3$. Proceeding as in the $P_{1}$ case, we see that the leading term in the expression for $a_{m, F}$ is

$$
\frac{7 p^{k}(p-1)}{3 x}
$$

As we did with $P_{1}$, we must now bound the number of fields $F$ with $\mathrm{G}(F / \mathbb{Q}) \cong P_{3}$ and $F / \mathbb{Q}$ ramified only at $p \infty$. As above, let $L=F^{a b}$, the degree 3 subfield of $\mathbb{Q}\left(\zeta_{p}\right)$. The field $L$ then admits a $Q_{8}$ extension $F_{0}$, which has 3 intermediate fields $L_{i}^{\prime}$, each of which is a $C_{2}$ extension of $L$, and unramified over $L$ by Lemma 2 . Let $K$ be the composite of the $L_{i}$. The field $L$ is totally real, so by the following lemma, $K$ is as well.

Lemma 7. Let $l$ be a totally real field and let $k / l$ be a Galois extension with group $Q_{8}$. Then the unique intermediate field that is degree 4 over $l$ is totally real (the field is unique because $Q_{8}$ has a unique subgroup of order 2).

Proof. Let $k^{\prime}=k \cap \mathbb{R}$. If $k^{\prime}=k$, we are done. Otherwise, $\left[k: k^{\prime}\right]=2$, and $k^{\prime}$ is the unique intermediate field of degree 4 over $l$.

Returning to the notation before Lemma 7, $K / L$ is unramified and $K$ is totally real, so the number of choices for $K$ is at most $r_{2,2}(k)$, where $k$ is the 2-rank of $\mathrm{Cl}(L)$. Using [14, Corollary 4], we have $|\mathrm{Cl}(L)|<\frac{1}{2} p$, so $k<\log _{2} p-1$. The field $F_{0}$ is a degree 2 extension of $K$, and

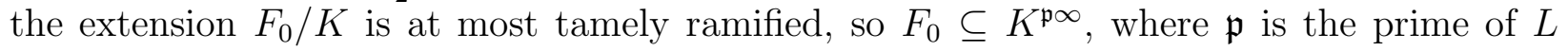
above $p$, viewed as an ideal of $K$. By class field theory, $\mathfrak{p}$ splits into a product of 4 primes in $K$ since it is principal in $L$. Using the exact sequence (2) applied to $\mathrm{Cl}^{p \infty}(K)$, we find that $\mathrm{G}\left(K^{\mathrm{p} \infty} / K\right)$ has 2-rank at most $4+12+$ the 2-rank of $\mathrm{Cl}(K)$. The order of $\mathrm{Cl}(K)$ is at most $\frac{22.2 p^{8}}{\pi^{12}}$ [15, page 95]. Set $\beta=\log _{2}\left(2^{12} \cdot \frac{22.2 p^{8}}{\pi^{12}}\right)$. If $F_{0} / K$ is unramified above $p$, there are at most

$$
r_{2,1}(\beta) r_{2,2}\left(\log _{2} p-1\right)
$$

choices for $F_{0}$, and if $F_{0} / K$ is ramified above $p$, there are at most

$$
\left(r_{2,1}(4+\beta)-r_{2,1}(\beta)\right) r_{2,2}\left(\log _{2} p-1\right) .
$$

The final step to bound the number of possible $F_{\rho}$ is to look at unramified $C_{3} \times C_{3}$ extensions of $F_{0}$ (which give our field $F_{\rho}$ ). This amounts to finding $r_{3,2}(l)$, where $l$ is the 3-rank of $\mathrm{Cl}\left(F_{0}\right)$. Using [15, page 95] and the fact that ramification in the infinite places does not contribute to the 3 -rank, we obtain

$$
l \leq \log _{3} \frac{22.2 p^{16}}{\pi^{24}}
$$


if $F_{0} / K$ is unramified, and

$$
l \leq \log _{3} \frac{22.2 p^{20}}{\pi^{24}}
$$

if $F_{0} / K$ is ramified.

We have $x=1$ or 2 , depending on whether $F_{0} / K$ is ramified. To obtain our bound for the number $a_{m}$ (where $m=3 k+3$ ) of $\rho$ ramified only at $p \infty$ with $G\left(F_{\rho} / \mathbb{Q}\right) \cong P_{3}$ and $N(\rho)=p^{m}$, we need to combine (8) with (9) and (11), and combine (8) with (10) and (12). Doing so, we find that the leading term of $a_{m}$ in $p$, which comes only from the $x=2$ case, is bounded by

$$
\frac{605134.5 p^{k+51}}{\pi^{50}}
$$

Finally, to conclude the section on $P_{3}$, we find that for the number $B_{m}$ of three-dimensional Artin representations with projective image $P_{3}$ and conductor dividing $p^{m}$, we have

Theorem 5. Notation as above,

$$
B_{m} \lesssim \frac{605134.5 p^{\frac{m}{3}+50}}{\pi^{50}}
$$

\section{Comparison to the two-dimensional case}

For comparison to the two-dimensional case, we look at [20]. Looking at Theorem 3 in [20] and multiplying by $\phi\left(p^{m}\right)=p^{m-1}(p-1)$ to account for all possible $\chi$, we see that the bounds for the number of two-dimensional representations with conductor $p^{m}$ and projective image isomorphic to $A_{4}$ or $S_{4}$ (and conjecturally for $A_{5}$ ) is on the order of $C p^{2 m-\epsilon} \log ^{4} p^{m}$ for $\epsilon=\frac{1}{6}, \frac{1}{8}$, or $\frac{1}{12}$ and $C$ a constant, both $\epsilon$ and $C$ depending on the projective image of the representation. Taking $p \rightarrow \infty$ for fixed $m$, we find that the bounds in [20] are better than our bounds for the imprimitive case. By going through the proofs of Theorems 4 and 5 in this paper, we may bound the number of $\rho$ with $\operatorname{Im} \pi \circ \rho=P_{1}, N(\rho) \mid p^{m}$, and with $\operatorname{Im} \pi \circ \rho=P_{3}, N(\rho) \mid p^{m}$, respectively, as $m \rightarrow \infty$ for fixed $p$. These bounds can be given by easily computable constants multiplied by the bounds given in Theorems 4 and 5 . Therefore, our bounds as $m \rightarrow \infty$ are stronger than those in [20], and our bounds are stronger as either $p$ or $m$ tends to $\infty$ for the primitive case. Of course, Wong's bounds hold for representations of any conductor $N$, not only for representations of prime power conductor.

Acknowledgements 1. The Author would like to thank his advisor, Joe Silverman, for his helpful conversations and Siman Wong for his correspondence concerning this work in relation to his own. The author is also appreciative of the referee's comments and suggestions, which were detailed and thoughtful.

\section{References}

[1] Gary Cornell, Joseph H. Silverman, and Glenn Stevens, editors. Modular forms and Fermat's last theorem. Springer-Verlag, New York, 1997. Papers from the Instructional 
Conference on Number Theory and Arithmetic Geometry held at Boston University, Boston, MA, August 9-18, 1995.

[2] Charles W. Curtis and Irving Reiner. Representation theory of finite groups and associative algebras. AMS Chelsea Publishing, Providence, RI, 2006. Reprint of the 1962 original.

[3] Pierre Deligne and Jean-Pierre Serre. Formes modulaires de poids 1. Ann. Sci. École Norm. Sup. (4), 7:507-530 (1975), 1974.

[4] Igor V. Dolgachev. Finite subgroups of the plane Cremona group. In Algebraic geometry in East Asia-Seoul 2008, volume 60 of Adv. Stud. Pure Math., pages 1-49. Math. Soc. Japan, Tokyo, 2010.

[5] W. Duke. The dimension of the space of cusp forms of weight one. Internat. Math. Res. Notices, (2):99-109, 1995.

[6] Leonard Evens. The Schur multiplier of a semi-direct product. Illinois J. Math., 16:166$181,1972$.

[7] Albrecht Fröhlich. Central extensions, Galois groups, and ideal class groups of number fields, volume 24 of Contemporary Mathematics. American Mathematical Society, Providence, RI, 1983.

[8] The GAP Group, GAP - Groups, Algorithms, and Programming, Version 4.6.2; 2013, (\protect \vrule width0pt \protect \href\{http://www.gap-system.org\}\{http://www.gap-syst

[9] Jing Long Hoelscher. Ray class groups of quadratic and cyclotomic fields. Int. J. Number Theory, 6(5):1169-1182, 2010.

[10] Bertram Huppert. Character theory of finite groups, volume 25 of de Gruyter Expositions in Mathematics. Walter de Gruyter \& Co., Berlin, 1998.

[11] I. Martin Isaacs. Character theory of finite groups. AMS Chelsea Publishing, Providence, RI, 2006. Corrected reprint of the 1976 original [Academic Press, New York; MR0460423].

[12] I. Martin Isaacs. Finite group theory, volume 92 of Graduate Studies in Mathematics. American Mathematical Society, Providence, RI, 2008.

[13] Chandrashekhar Khare. Remarks on $\bmod p$ forms of weight one. Internat. Math. Res. Notices, (3):127-133, 1997.

[14] Stéphane R. Louboutin. Upper bounds for residues of Dedekind zeta functions and class numbers of cubic and quartic number fields. Math. Comp., 80(275):1813-1822, 2011.

[15] Hyunsuk Moon. The number of monomial mod $p$ Galois representations with bounded conductor. Tohoku Math. J. (2), 55(1):89-98, 2003. 
[16] L. B. Pierce. The 3-part of class numbers of quadratic fields. J. London Math. Soc. (2), 71(3):579-598, 2005.

[17] J.-P. Serre. Modular forms of weight one and Galois representations. In Algebraic number fields: L-functions and Galois properties (Proc. Sympos., Univ. Durham, Durham, 1975), pages 193-268. Academic Press, London, 1977.

[18] Jean-Pierre Serre. Linear representations of finite groups. Springer-Verlag, New York, 1977. Translated from the second French edition by Leonard L. Scott, Graduate Texts in Mathematics, Vol. 42.

[19] Jean-Pierre Serre. Local fields, volume 67 of Graduate Texts in Mathematics. SpringerVerlag, New York, 1979. Translated from the French by Marvin Jay Greenberg.

[20] Siman Wong. Automorphic forms on GL(2) and the rank of class groups. J. Reine Angew. Math., 515:125-153, 1999. 